\title{
Article \\ Percutaneous Core Needle Biopsy Can Efficiently and Safely Diagnose Most Primary Bone Tumors
}

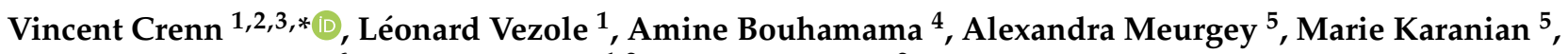 \\ Perrine Marec-Bérard ${ }^{6}$, François Gouin ${ }^{1,2}$ and Gualter Vaz ${ }^{2}$ \\ 1 Orthopedics and Trauma Department, University Hospital Hotel-Dieu, CHU Nantes, 44000 Nantes, France; \\ lvezole@gmail.com (L.V.); francois.gouin@unicancer.lyon.fr (F.G.) \\ 2 Département de Chirurgie, Centre de Lutte Contre le Cancer Léon Bérard, 69008 Lyon, France; \\ gualter.vaz@unicancer.lyon.fr \\ 3 PhyOs 1238, INSERM, Nantes University, UMR1238 Phy-Os “Bone Sarcomas and Remodeling of Calcified \\ Tissues", 44035 Nantes, France \\ 4 Département de Radiologie, Centre de Lutte Contre le Cancer Léon Bérard, 69008 Lyon, France; \\ Amine.BOUHAMAMA@lyon.unicancer.fr \\ 5 Département d'anatomopathologie, Centre de Lutte Contre le Cancer Léon Bérard, 69008 Lyon, France; \\ Alexandra.MEURGEY@lyon.unicancer.fr (A.M.); marie.karanian@lyon.unicancer.fr (M.K.) \\ 6 Département d'oncologie Pédiatrique, Centre de Lutte Contre le Cancer Léon Bérard, 69008 Lyon, France; \\ perrine.marec-berard@ihope.fr \\ * Correspondence: vincent.crenn@chu-nantes.fr
}

check for updates

Citation: Crenn, V.; Vezole, L.; Bouhamama, A.; Meurgey, A.; Karanian, M.; Marec-Bérard, P.; Gouin, F.; Vaz, G. Percutaneous Core Needle Biopsy Can Efficiently and Safely Diagnose Most Primary Bone Tumors. Diagnostics 2021, 11, 1552. https://doi.org/10.3390/

diagnostics11091552

Academic Editor: Dario Marchetti

Received: 3 August 2021

Accepted: 26 August 2021

Published: 27 August 2021

Publisher's Note: MDPI stays neutral with regard to jurisdictional claims in published maps and institutional affiliations.

Copyright: (C) 2021 by the authors Licensee MDPI, Basel, Switzerland. This article is an open access article distributed under the terms and conditions of the Creative Commons Attribution (CC BY) license (https:/ / creativecommons.org/licenses/by/ $4.0 /)$.

\begin{abstract}
A biopsy is a prerequisite for the diagnosis and evaluation of musculoskeletal tumors. It is considered that surgical biopsy provides a more reliable diagnosis because it can obtain more tumor material for pathological analysis. However, it is often associated with a significant complication rate. Imaging-guided percutaneous core needle biopsy (PCNB) is now widely used as an alternative to surgical biopsy; it appears to be minimally invasive, possibly with lower complication rates. This study evaluates the diagnostic yield of the preferred use of PCNB in a referral center, its accuracy, and its complication rate. The data relating to the biopsy and the histological analysis were extracted from the database of a bone tumor reference center where PCNB of bone tumors was discussed as a first-line option. 196 bone tumors were biopsied percutaneously between 2016 and 2020. They were located in the axial skeleton in $21.4 \%$ (42) of cases, in the lower limb in $58.7 \%$ (115), and in the upper limb in $19.9 \%$ (39) cases. We obtained a diagnosis yield of $84.7 \%$ and a diagnosis accuracy of $91.7 \%$. The overall complication rate of the percutaneous biopsies observed was $1.0 \%(n=2)$, consisting of two hematomas. PCNB performed in a referral center is a safe, precise procedure, with a very low complication rate, and which avoids the need for first-line open surgical biopsy. The consultation between pathologist, radiologist, and clinician in an expert reference center makes this technique an effective choice as a first-line diagnosis tool.
\end{abstract}

Keywords: diagnostic accuracy; percutaneous biopsy; bone sarcoma

\section{Introduction}

The incidence of primitive bone tumors is low, but they most commonly affect young adults. After a complete radiological examination, the great majority of bone tumors require a biopsy to determine whether they are benign or malignant, after which the appropriate therapeutic intervention can be determined. Surgical open biopsies are historically considered the gold standard in musculoskeletal tumor diagnosis as they offer the best diagnostic accuracy, around $98 \%$, according to research [1,2]. Nevertheless, the performance of open biopsy comes with morbidity consequences [3], a high cost, and potential logistical difficulties that can delay its realization $[4,5]$.

Recent studies have shown that percutaneous core needle biopsy (PCNB) results in a lower complication rate (0-2\%) [3] than open biopsy $(16 \%)[1,6]$; it also results in shorter 
hospital stays and lower costs while maintaining high diagnostic accuracy. Its accuracy rates for bone tumors are not clearly defined and vary from 66 to $98 \%[4,6,7]$, with a higher diagnostic yield for bone lesions than for soft tissue lesions [8].

In this context, our hypothesis is that core needle biopsy carried out on primary bone tumors, when realized under optimal conditions in a musculoskeletal sarcoma reference center, offers a reliable diagnostic tool. This study evaluates the diagnostic yield and accuracy of first-line radio-guided PCNB in a referral center, as well as its complication rates.

\section{Materials and Methods}

\subsection{Study Design}

This retrospective monocentric study included all patients who underwent a diagnostic PCNB for a suspected bone tumor as initial care at the Centre Léon Bérard (CLB) referral musculoskeletal sarcoma center between 2016 and 2020. We assessed patient records for clinical context, tumor location, biopsy modalities and results, and complications following the procedure. This study was approved by the institutional research ethics committee. Cases of PCNB associated with radiological treatment (such as cryotherapy or ultra-sonotherapy) were excluded from this analysis. Solid metastatic tumor diagnoses were also excluded. A previous biopsy or prior diagnosis data were considered exclusion criteria as we focused on first-line diagnosis.

\subsection{Biopsy Protocol}

Percutaneous core needle biopsy of bone tumors was discussed as a first-line option in our therapeutic structure, and it was performed by experienced skeletal radiologists. Biopsy indication, choice between techniques, and anesthesia modalities were discussed in a weekly multidisciplinary team meeting. General anesthesia was preferred for young patients, or for complex and deep biopsy techniques requiring immobilization. When PCNB was validated, the needle tract was systematically approved after discussion between the interventional radiologist and the orthopedic surgeon.

Before each procedure, coagulation status was verified, and premedication was administered $30 \mathrm{~min}$ prior to the biopsy. The PCNBs were performed under CT-scan (Figure 1) (SOMATOM Emotion 16; Siemens Healthcare, Erlangen, Germany) or ultrasound (Aplio 500; Toshiba Medical Systems, Tokyo, Japan) guidance. For extra-osseous extension, a 13G coaxial needle biopsy (BARD Biopsy Systems, Crawley, UK) was inserted into the tumor and four to ten core needle biopsies were obtained using a 14G Tru-Cut biopsy needle (BARD Biopsy Systems, Crawley, UK). For intra-osseous lesions, we used an Arrow OnControl 13- or 11-gauge bone-access electric-powered device (Vidacare, Shavano Park, TX, USA). For osteoblastic lesions with no extra-osseous extension, we used an Arrow OnControl co-axial 12-gauge bone-access electric-powered device (Vidacare, Shavano Park, TX, USA), and two to three trephine biopsies were obtained using a 13G Arrow OnControl trephine. For osteolytic lesions, the same procedure was performed but four additional core needle biopsies were obtained using the $14 \mathrm{G}$ Tru-Cut biopsy needle. To allow further resection of the biopsy tract, the cutaneous needle entry point was tattooed with sterile China ink (Pigment RADSAFE noir; Biotic Phocea, Marseille, France) using MICROLET lancets (Bayer Healthcare, Neuilly-sur-Seine, France).

\subsection{Outcome Measure}

We evaluated the diagnostic yield, which was calculated in order to assess whether core needle biopsy can provide diagnostic information adequately. The pathology reports on all the biopsies were reviewed and categorized by pathologists as diagnostic and nondiagnostic specimens, or as insufficient specimens for pathological diagnosis. Patients who needed a second biopsy were also considered non-diagnostic. Patients who required an open biopsy or a second $\mathrm{PCNB}$ in order to obtain a final diagnosis were recorded. We determined the diagnostic accuracy after the first PCNB; accurate percutaneous biopsy results were considered to be those that were in agreement with the subsequent pathological 
examination results from the surgical specimens regarding the tumor's histological nature (malignant or benign). Complications (i.e., hematoma, infection, fracture) in the month following biopsy were reported.
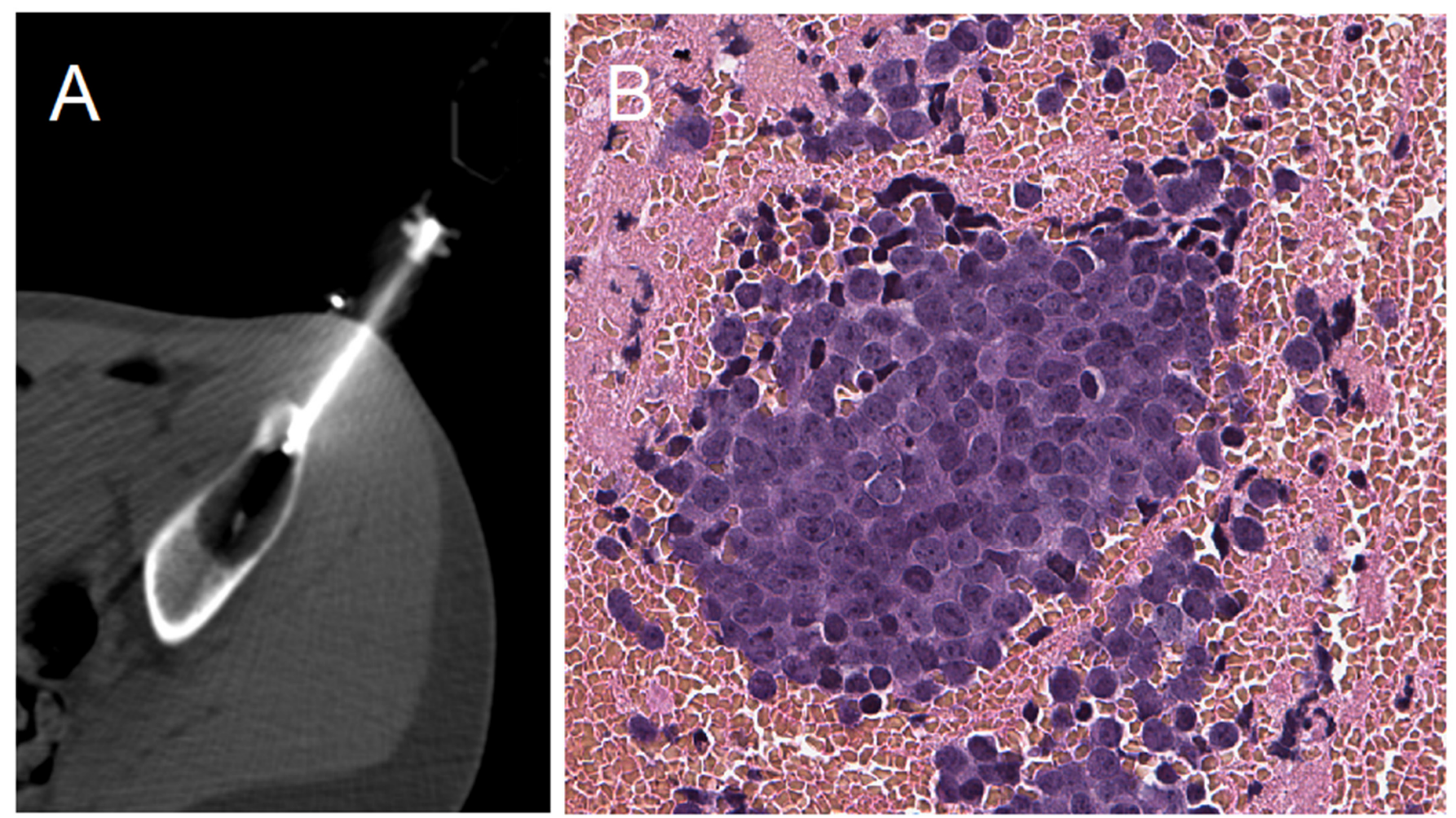

Figure 1. Percutaneous core needle biopsy CT-scan view and microscopic histological view. (A): PCNB CT-Scan axial view of a supra-acetabular iliac lytic aggressive lesion biopsy. (B): The final diagnosis of a Ewing sarcoma on a histopathological section, $\times 200$ magnification.

\subsection{Statistics and Ethics}

The data relating to the biopsy and the histological analysis were extracted from the database of a reference center for the management of musculoskeletal tumors where the PCNB of bone tumors was discussed as a first-line option. Data analyses were performed using SPSS in order to assess diagnostic parameters and associated results.

\section{Results}

\subsection{Study Population}

The study included 196 patients who underwent a core needle percutaneous diagnostic biopsy for a suspected bone tumor (Figure 2) (Table 1). Most PCNBs were performed under CT-Scan guidance $(n=182,92.9 \%)$. Local anesthesia was used for $91.8 \%$ of patients $(n=180)$. For patients under 18 years old, local anesthesia was used for $75.0 \%(n=9 / 12)$. 


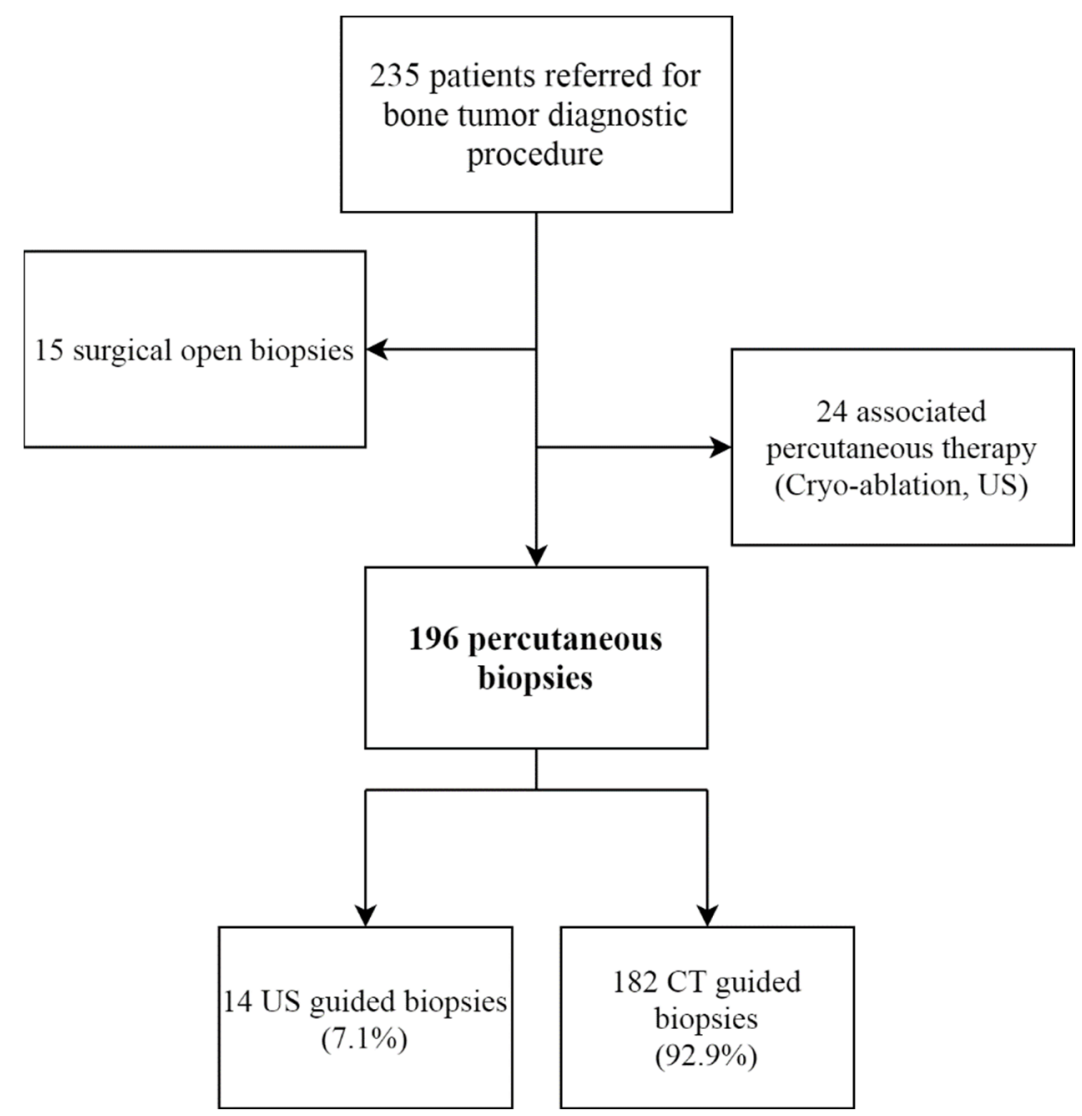

Figure 2. Study flow-chart. US: ultrasound; CT: computed tomography.

Table 1. Study population data.

\begin{tabular}{|c|c|}
\hline & Study Cohort $(n=196)$ \\
\hline Age & $41.9(8-90)$ \\
\hline \multicolumn{2}{|l|}{ Sex } \\
\hline Female & $106(54.1 \%)$ \\
\hline Male & $90(45.9 \%)$ \\
\hline \multicolumn{2}{|l|}{ Performance status } \\
\hline 0 & $154(78.6 \%)$ \\
\hline $1-2$ & $37(18.9 \%)$ \\
\hline $3-4$ & $5(2.5 \%)$ \\
\hline \multicolumn{2}{|l|}{ Localization } \\
\hline Upper limb & $39(19.9 \%)$ \\
\hline Lower limb & $115(58.7 \%)$ \\
\hline Axial skeleton & $42(21.4 \%)$ \\
\hline \multicolumn{2}{|l|}{ Biopsy type } \\
\hline \multicolumn{2}{|l|}{ Percutaneous guided } \\
\hline Echography & $14(7.1 \%)$ \\
\hline $\mathrm{CT}$ & $182(92.9 \%)$ \\
\hline \multicolumn{2}{|l|}{ Anesthesiology modality } \\
\hline Local anesthesia & $180(91.8 \%)$ \\
\hline General anesthesia & $16(8.2 \%)$ \\
\hline
\end{tabular}

The final diagnosis revealed a malignant tumor in $49.5 \%$ of cases $(n=97)$, mostly osteosarcoma (13.8\%), chondrosarcoma (13.8\%), and Ewing sarcoma $(10.2 \%)$. The most frequent benign lesion was a giant cell tumor (GCT) $(n=19 ; 9.7 \%)$ (Table 2$)$. 
Table 2. Final histopathology results for bone tumors. *: no specific diagnosis, but no atypical cells nor malignancy detected.

\begin{tabular}{|c|c|c|c|}
\hline \multirow{2}{*}{$\begin{array}{c}\text { Sampling Outcome Diagnosis } \\
\text { Malignant }\end{array}$} & \multicolumn{3}{|c|}{ Final Diagnosis } \\
\hline & & Begnin & \\
\hline Osteosarcoma & $27(13.8 \%)$ & Giant cell tumor & $19(9.7 \%)$ \\
\hline Chondrosarcoma & $27(13.8 \%)$ & No malignancy lesion * & $18(9.2 \%)$ \\
\hline Ewing sarcoma & $20(10.2 \%)$ & Angioma & $7(3.6 \%)$ \\
\hline Lymphoma & $11(5.6 \%)$ & Chondroblastoma & $7(3.6 \%)$ \\
\hline Chordoma & $4(2.0 \%)$ & Osteoid Osteoma & $5(2.6 \%)$ \\
\hline Myeloma or plasmacytoma & $4(2.0 \%)$ & Nonossifying fibroma & $5(2.6 \%)$ \\
\hline High-grade sarcoma (NOS) & $1(0.5 \%)$ & Langerhans cell histiocytosis & $5(2.6 \%)$ \\
\hline Pleomorphic cell sarcoma & $1(0.5 \%)$ & Osteochondroma or osteoma & $4(2.0 \%)$ \\
\hline Adamantinoma & $1(0.5 \%)$ & Fibrous dysplasia & $4(2.0 \%)$ \\
\hline \multirow[t]{13}{*}{ Malignant hemangioendothelioma } & $1(0.5 \%)$ & Infection & $3(1.5 \%)$ \\
\hline & & Simple bone cyst & $3(1.5 \%)$ \\
\hline & & Benign fibrous histiocytoma & $3(1.5 \%)$ \\
\hline & & Chondroma & $2(1.0 \%)$ \\
\hline & & Osteoblastoma & $2(1.0 \%)$ \\
\hline & & Reactive bone changes & $2(1.0 \%)$ \\
\hline & & Paget's disease & $2(1.0 \%)$ \\
\hline & & Begnin hemangioma & $2(1.0 \%)$ \\
\hline & & Atypic cartilaginous tumor & $1(0.5 \%)$ \\
\hline & & Brown tumor & $1(0.5 \%)$ \\
\hline & & Intra-osseous lipoma & $1(0.5 \%)$ \\
\hline & & Gout tophus & $1(0.5 \%)$ \\
\hline & & Chondrocalcinosis & $1(0.5 \%)$ \\
\hline Total & $97(49.5 \%)$ & & $99(51.5 \%)$ \\
\hline
\end{tabular}

\subsection{Diagnostic Yield}

The diagnostic yield after the first PCNB was obtained in $84.7 \%$ of cases, allowing a diagnosis and clear therapeutic strategy for 166 patients (Figure 3). For 30 patients $(15.3 \%)$, no diagnostic certainty was observed; in two cases this was due to a technical failure to harvest sufficient tissue, and in the other cases (28 cases) the bone biopsy did not make it possible to obtain a final diagnostic certainty for diverse reasons (such as necrotic tissue, non-specific tissue or diagnostic doubt). Of these patients, $17(8.7 \%)$ obtained a complementary biopsy, 6 of whom received an open surgical biopsy (3.1\%); the remaining 11 received a second PCNB (5.6\%). Other cases were considered benign lesions with no precise diagnosis (absence of malignant cells) after discussion in multidisciplinary team meetings, and were followed up radiographically. A third-line biopsy was performed surgically in an open fashion in three cases after an ineffective second-line PCNB. 


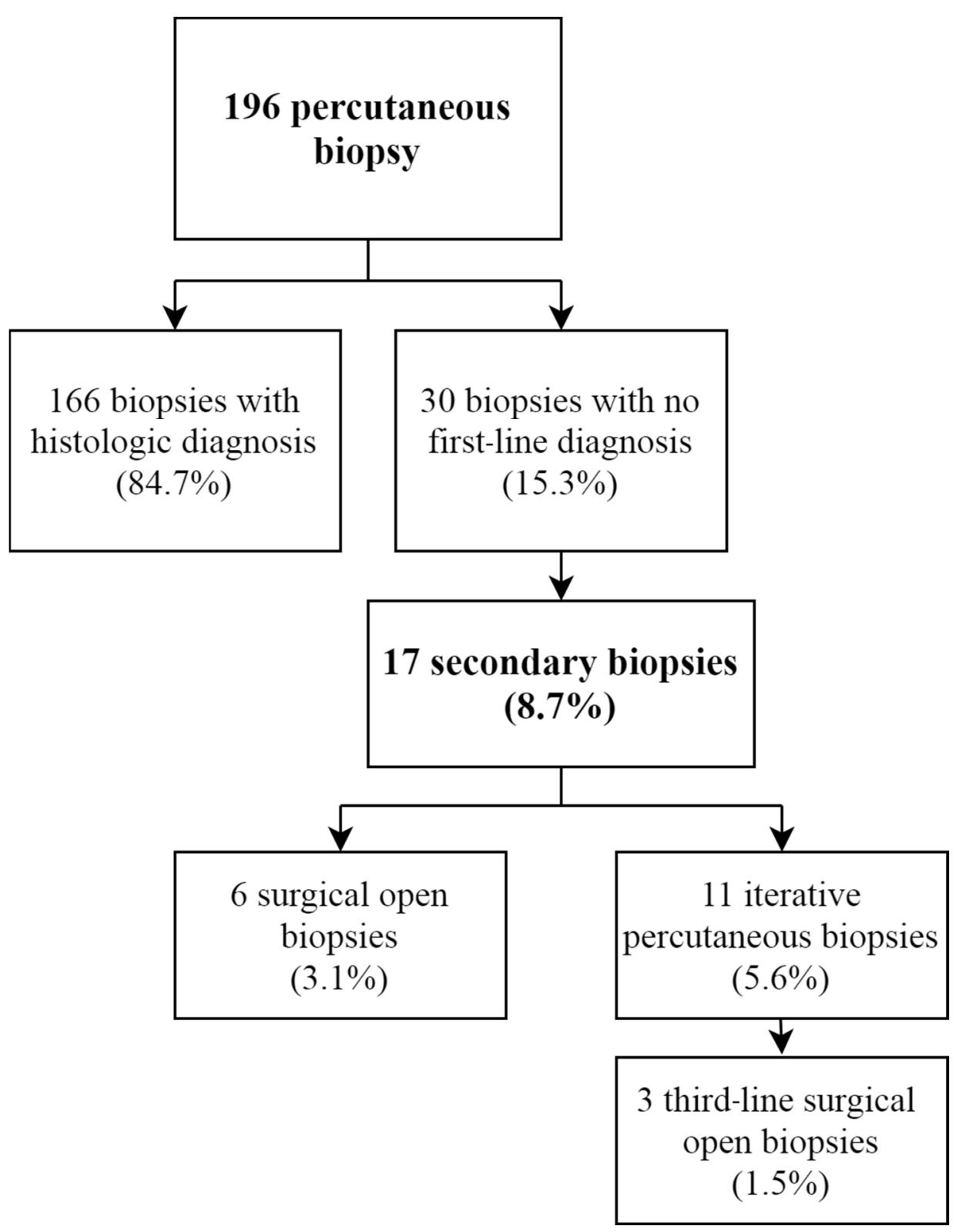

Figure 3. Percutaneous biopsy yield flow-chart.

\subsection{Diagnostic Accuracy}

We compared the first diagnosis proposed following PCNB with the definitive diagnosis obtained from the surgical specimen of 133 operated patients $(67.8 \%)$. We obtained a PCNB accuracy of $91.7 \%(n=122 / 133)$. Of the eleven remaining diagnoses with a discrepancy, eight were unable to obtain a formal initial diagnosis $(72.7 \%)$. Second-line biopsies were performed for seven of the eleven diagnoses with a discrepancy (63.6\%). Five of these were surgical open biopsies (45.4\%) and two were CT-guided micro-biopsies (18.2\%).

The second-line biopsies performed on these seven patients made it possible to obtain a final diagnosis in six cases, increasing the diagnostic accuracy to $96.2 \%(128 / 133)$.

Focusing on malignant lesion discrepancies, second-line biopsies made it possible to identify three malignant lesions (two osteosarcomas and one malignant hemangioendothelioma) (Figure 4). These were confirmed by surgical resection specimens, except for one chondroblastic osteosarcoma diagnosed with a second-line PCNB, which turned out to be a conventional chondrosarcoma, according to the final surgical diagnosis. One undifferentiated pleomorphic sarcoma of the bone on initial biopsy was an osteosarcoma, according to the surgical specimen. Finally, one initial diagnosis of chondroma was directly 
operated on after PCNB and was finally characterized as a chondrosarcoma, according to the surgical specimen. This was the only example of a discrepancy between benign to malignant, excluding secondary rectified diagnoses.

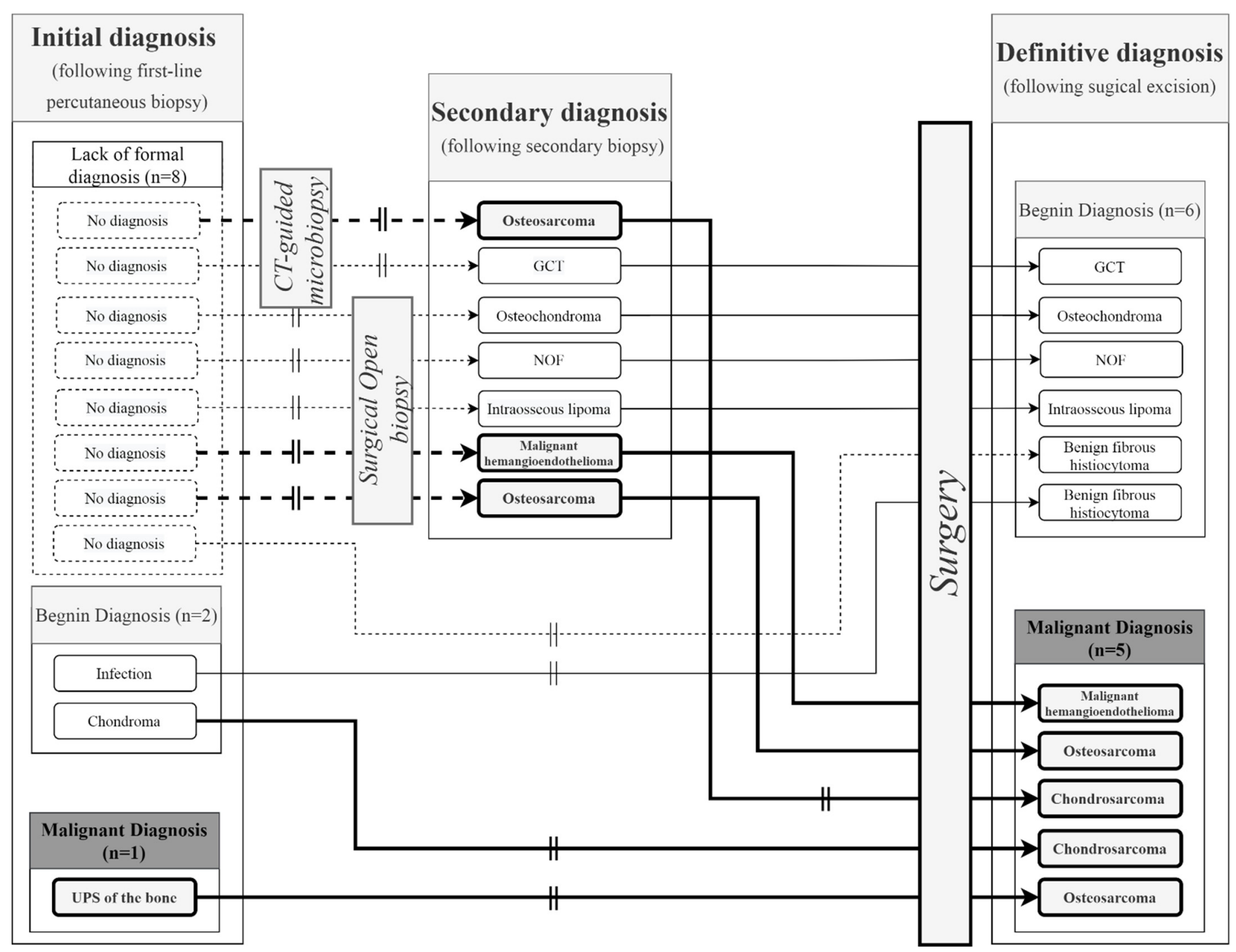

Figure 4. Focus on the 11 diagnostic discrepancies. The vertical double lines represent diagnostic discrepancy. The bold arrows represent patients with a final malignant diagnosis following a second biopsy (open or CT-guided) or direct surgery. The dotted arrows represent initial non-informative micro-biopsies with no diagnosis. GCT: Giant cell tumor; NOF: Non-ossifying fibroma.

\subsection{Complications}

We observed a complication rate of $1.0 \%$, consisting of only two hematomas, with no need for any treatment other than ice and medical surveillance. Neither infections nor fractures were observed among the cohort during the one-month follow-up.

\section{Discussion}

Until recently, open surgical biopsy was considered the gold standard for bone tumor diagnosis. Nonetheless, the inherent cost and iatrogenicity of open biopsy, balanced by considerable progress in histological characterization, are moving the cursor towards PCNB as a first-line diagnosis tool for skeletal tumors, with evolving reference guidelines [9]. Our cohort confirmed this recent trend, as we obtained a high diagnostic accuracy of $91.7 \%$, associated with an $84.7 \%$ diagnostic yield. Moreover, no complications, apart from two cases of hematoma $(1.0 \%)$, were observed using the PCNB technique. 


\subsection{Diagnostic Yield}

In our experience, this technique yielded sufficient amounts of tissue to make a diagnosis in $84.7 \%$ of cases, with the need for a second biopsy in $8.7 \%$ of cases. The diagnostic yield seems acceptable, and a secondary PCNB might be easily proposed in the case of a non-contributive biopsy $(n=11,5.6 \%)$ or a lack of certainty. Complementary surgical open biopsy was necessary in nine cases $(4.6 \%)$, as a second-line $(n=6,3.1 \%)$ or third-line ( $n=3,1.5 \%$ ) diagnostic tool. Most of the uncertain diagnoses consisted of benign lesions with no need for surgery and were simply followed up radiologically; this consideration is corroborated by the high diagnostic accuracy observed for bone tumors requiring a surgical excision strategy. These findings are in line with the conclusions in the meta-analysis by Kubo et al., which suggest that a first-line core needle biopsy should be performed by expert radiologists, and that a surgical biopsy should be performed secondarily if diagnosis following the core needle biopsy does not match the clinical presentation and radiographic findings [10].

\subsection{Diagnostic Accuracy}

The accuracy of PCNB for operated patients with a definitive histological diagnosis from a surgical specimen is a methodologically high-value criterion. For this specific outcome, we obtained a 91.7\% diagnostic accuracy. Moreover, six (54.5\%) of the initial no-diagnoses were completed successfully with a second-line biopsy, increasing diagnostic accuracy to $96.2 \%$. These results are consistent with recent research on this subject: in bone and soft tissue tumors, Yang et al. achieved a diagnostic accuracy of $89 \%$ in 509 cases [11]; Klein et al. observed $83.6 \%$ in their study [12].

It is also of interest to consider that two of the five malignant discrepancies $(40.0 \%)$ were due to a cartilaginous tumor, which presents well-known difficulties in bone tumor diagnosis. In fact, for this specific histological type, it was possible to confirm only the chondral nature of the lesion using PCNB and not its aggressiveness, as bone resorption images are absent on these samples most of the time [9]. Chondral tumors specifically require a multidisciplinary approach for accurate diagnosis, associating multimodal imaging analysis, as stated by Sharif et al. [13]. Moreover, these data should also be correlated with the clinical presentation, and it is only under these circumstances that the tumor board can propose a therapeutic strategy [14].

We observed only one event $(0.8 \%)$ with a discrepancy in which the diagnosis went from benign to malignant, excluding rectified diagnoses on second biopsy. Within this criterion, our findings are in line with previous studies confirming the reliability of PCNB for differentiating between benign and malignant lesions, even in aggressive radiolucent bone tumors $[15,16]$.

\subsection{Complications}

One of the major points of interest regarding the use of PCNB in bone tumor diagnosis remains its low rate of complications. Our cohort confirmed this tendency with a rate of complications of one per cent $(1.0 \%, n=2)$, consisting only of two hematomas, with no need for surgical reoperation. This exceptionally low rate compared to that of open biopsy, which is up to $16 \%[17,18]$, is of great interest, as an infection or a fracture following a biopsy can drastically worsen the prognosis for bone sarcoma.

\subsection{Limitations and Strengths}

Some of the patients in the cohort were not included in the study, such as those who had a direct open biopsy or an associated therapeutic treatment. Moreover, as a consequence of the retrospective design, it was not always possible to precisely obtain the decision process that guided the second-line biopsy towards an open biopsy or PCNB.

As proposed by some authors [4], we also decided to not consider in the precision parameter calculation any patients with no surgical confirmation of their diagnosis, as our 
analyses were based on histological accuracy. We decided that it would be inaccurate to confirm or deny a precise histological diagnosis based only on patient follow-up.

This work, carried out in a specialized sarcoma center, is of interest because of its cohort size and homogeneity, focusing especially on bone tumors. In this context, it seems important to remember that a key factor for external validation and the appropriate use of PCNB is an experienced musculoskeletal tumor team that communicates frequently and is capable of correlating clinical, radiographic, and histological information for each patient.

\section{Conclusions}

Percutaneous core needle biopsy with radio-guided drilling performed in a referral center is a safe, precise procedure, with a low complication rate, that prevents the need for a first-line open surgical biopsy. Even though its yield is lower than that of open biopsy, its accuracy concerning operated lesions remains at a high level, at 91.7\%. Moreover, a second-line biopsy in cases with a lack of certainty raises the diagnostic accuracy to $96.2 \%$. Concerted work with anatomo and pathology teams in expert reference centers makes this technique an effective choice as a first-line diagnostic tool.

Author Contributions: Conceptualization, G.V. and F.G.; methodology, G.V. and F.G.; formal analysis, V.C.; investigation, A.B., A.M., M.K., P.M.-B., F.G. and G.V.; data curation, L.V. and V.C.; writingoriginal draft preparation, V.C. and L.V.; writing-review and editing, V.C., L.V., A.B., A.M., M.K., P.M.-B., F.G. and G.V.; supervision, F.G. and G.V. All authors have read and agreed to the published version of the manuscript.

Funding: This research received no external funding.

Institutional Review Board Statement: This study was conducted according to the guidelines of the Declaration of Helsinki, and approved by the Centre de Lutte Contre le Cancer Léon Bérard Institutional Review board. Ethical review and approval were waived for this study, due to it retrospective design.

Informed Consent Statement: Informed consent was obtained from all subjects involved in the study.

Data Availability Statement: The datasets generated and/or analyzed during this study are available from the corresponding author on reasonable request.

Conflicts of Interest: The authors declare no conflict of interest.

\section{References}

1. Le, H.B.Q.; Lee, S.T.; Munk, P.L. Image-Guided Musculoskeletal Biopsies. Semin. Interv. Radiol. 2010, 27, 191-198. [CrossRef]

2. Srisawat, P.; Veeraphun, P.; Punyaratabandhu, T.; Chareonvareekul, S.; Songpattanasilpa, T.; Sritanabutr, P.; Pipithkul, S. Comparative Study of Diagnostic Accuracy between Office-Based Closed Needle Biopsy and Open Incisional Biopsy in Patients with Musculoskeletal Sarcomas. J. Med. Assoc. Thai 2014, 97 (Suppl. 2), S30-S38. [PubMed]

3. Sung, K.-S.; Seo, S.-W.; Shon, M.-S. The Diagnostic Value of Needle Biopsy for Musculoskeletal Lesions. Int. Orthop. 2009, 33, 1701-1706. [CrossRef] [PubMed]

4. Ceraulo, A.; Ouziel, A.; Lavergne, E.; Perrier, L.; Decouvelaere, A.-V.; Chotel, F.; Thiesse, P.; Marec-Berard, P. Percutaneous Guided Biopsy for Diagnosing Suspected Primary Malignant Bone Tumors in Pediatric Patients: A Safe, Accurate, and Cost-Saving Procedure. Pediatr. Radiol. 2017, 47, 235-244. [CrossRef] [PubMed]

5. Skrzynski, M.C.; Biermann, J.S.; Montag, A.; Simon, M.A. Diagnostic Accuracy and Charge-Savings of Outpatient Core Needle Biopsy Compared with Open Biopsy of Musculoskeletal Tumors. J. Bone Jt. Surg. Am. 1996, 78, 644-649. [CrossRef] [PubMed]

6. Filippiadis, D.K.; Charalampopoulos, G.; Mazioti, A.; Keramida, K.; Kelekis, A. Bone and Soft-Tissue Biopsies: What You Need to Know. Semin. Interv. Radiol. 2018, 35, 215-220. [CrossRef]

7. Rimondi, E.; Rossi, G.; Bartalena, T.; Ciminari, R.; Alberghini, M.; Ruggieri, P.; Errani, C.; Angelini, A.; Calabrò, T.; Abati, C.N.; et al. Percutaneous CT-Guided Biopsy of the Musculoskeletal System: Results of 2027 Cases. Eur. J. Radiol. 2011, 77, 34-42. [CrossRef] [PubMed]

8. Pouedras, M.; Briand, S.; Crenn, V.; Cassagnau, E.; Gouin, F. Non Image-Guided Core Needle Biopsies Can Be Used Safely to Improve Diagnostic Efficiency for Soft Tissue Tumors. Surg. Oncol. 2021, 37, 101518. [CrossRef] [PubMed]

9. Galant, C.; Bouvier, C.; Larousserie, F.; Aubert, S.; Audard, V.; Brouchet, A.; Marie, B.; Guinebretière, J.-M.; de Pinieux du Bouexic, G. Diagnostic histologique des tumeurs osseuses: Biopsie chirurgicale ou biopsie percutanée? Recommandations des pathologistes du réseau de référence des tumeurs osseuses (RESOS). Bull. Du Cancer 2018, 105, 368-374. [CrossRef] [PubMed] 
10. Kubo, T.; Furuta, T.; Johan, M.P.; Sakuda, T.; Ochi, M.; Adachi, N. A Meta-Analysis Supports Core Needle Biopsy by Radiologists for Better Histological Diagnosis in Soft Tissue and Bone Sarcomas. Med. Baltim. 2018, 97, e11567. [CrossRef] [PubMed]

11. Yang, J.; Frassica, F.J.; Fayad, L.; Clark, D.P.; Weber, K.L. Analysis of Nondiagnostic Results after Image-Guided Needle Biopsies of Musculoskeletal Lesions. Clin. Orthop. Relat. Res. 2010, 468, 3103-3111. [CrossRef] [PubMed]

12. Klein, A.; Fell, T.; Birkenmaier, C.; Fromm, J.; Jansson, V.; Knösel, T.; Dürr, H.R. Relative Sensitivity of Core-Needle Biopsy and Incisional Biopsy in the Diagnosis of Musculoskeletal Sarcomas. Cancers 2021, 13, 1393. [CrossRef] [PubMed]

13. Sharif, B.; Lindsay, D.; Saifuddin, A. The Role of Imaging in Differentiating Low-Grade and High-Grade Central Chondral Tumours. Eur. J. Radiol. 2021, 137, 109579. [CrossRef] [PubMed]

14. Roitman, P.D.; Farfalli, G.L.; Ayerza, M.A.; Múscolo, D.L.; Milano, F.E.; Aponte-Tinao, L.A. Is Needle Biopsy Clinically Useful in Preoperative Grading of Central Chondrosarcoma of the Pelvis and Long Bones? Clin. Orthop. Relat. Res. 2017, 475, 808-814. [CrossRef] [PubMed]

15. Hegde, V.; Burke, Z.D.C.; Park, H.Y.; Zoller, S.D.; Johansen, D.; Kelley, B.V.; Levine, B.; Motamedi, K.; Federman, N.C.; Seeger, L.L.; et al. Is Core Needle Biopsy Reliable in Differentiating Between Aggressive Benign and Malignant Radiolucent Bone Tumors? Clin. Orthop. Relat. Res. 2018, 476, 568-577. [CrossRef] [PubMed]

16. Taupin, T.; Decouvelaere, A.-V.; Vaz, G.; Thiesse, P. Accuracy of Core Needle Biopsy for the Diagnosis of Osteosarcoma: A Retrospective Analysis of 73 patients. Diagn. Interv. Imaging 2016, 97, 327-331. [CrossRef] [PubMed]

17. Mankin, H.J.; Mankin, C.J.; Simon, M.A. The Hazards of the Biopsy, Revisited. Members of the Musculoskeletal Tumor Society. J. Bone Jt. Surg. Am. 1996, 78, 656-663. [CrossRef] [PubMed]

18. Errani, C.; Traina, F.; Perna, F.; Calamelli, C.; Faldini, C. Current Concepts in the Biopsy of Musculoskeletal Tumors. Sci. World J. 2013, 2013, 538152. [CrossRef] [PubMed] 